\title{
Lean Maturity, Lean Sustainability
}

\author{
Frances Jørgensen, Rikke Matthiesen, Jacob Nielsen and John Johansen \\ Center for Industrial Production Aalborg University Fibigerstrade 169220 \\ Aalborg E., Denmark \\ corresponding author: frances@production.aau.dk
}

\begin{abstract}
Although lean is rapidly growing in popularity, its implementation is far from problem free and companies may experience difficulties sustaining long term success. In this paper, it is suggested that sustainable lean requires attention to both performance improvement and capability development. A framework for describing levels of lean capability is presented, based on a brief review of the literature and experiences from 12 Danish companies currently implementing lean. Although still in its emerging phase, the framework contributes to both theory and practice by describing developmental stages that support lean capability development and consequently, lean sustainability.
\end{abstract}

\section{Keywords}

Lean Capability Model, Lean Sustainability, Lean Manufacturing

\section{Introduction}

During recent years lean as a process management philosophy has rapidly gained popularity in the manufacturing sector, and in some countries, in both private and public administrative and service organizations as well. With its focus on just-intime production, elimination of waste, and continuous improvement, many companies are reporting impressive performance gains [1]. According to Womack et al. [2], lean also provides opportunities for a positive and fulfilling working environment for employees, due the employees' involvement in and ownership of problem-solving and improvement activities, more diversified work functions requiring varied skills and abilities, and increased cross-functional and interorganizational functions.

A number of empirical studies have shown, however, that implementation of lean may be anything but a positive experience for employees [3] and studies by Landsbergies et al. [4] suggest that the increased intensity and higher degree of 
standardization of the work functions associated with lean may have detrimental effects on employees, both psychologically and physically. These negative consequences on the workforce may be aggravated by a skewed focus on the implementation of lean tools and methods that sacrifice the development of a lean culture [5]. In the most extreme cases, companies may become almost obsessed with cost and/or staff reduction that the work becomes "more intense, stressful and hazardous" [6], thereby trading potential long term, sustainable improvement for short term gains, sometimes even leading to what is referred to as organizational anorexia [7]. More common are situations in which companies have difficulty maintaining momentum for lean after often dramatic performance improvements are realized following the initial implementation of lean. In other words, companies have difficulties sustaining lean over time.

One explanation for the difficulties companies encounter in sustaining lean may be attributed to a lack of focus on the developmental progression of lean capabilities amongst the members of the organization. By focusing on developing lean capabilities, members of the organization should then become progressively better at doing lean while at the same time, creating a learning environment that supports a lean culture. The objective of this paper is to present a framework describing the development of lean capabilities towards a state of maturity conducive to long term sustainability of lean. The framework is derived from the current lean literature and descriptions of lean experiences in 12 large Danish organizations currently involved in a lean implementation process. In the next sections, the sustainability as it relates to lean is introduced; thereafter, the development of the concept of lean is discussed as the foundation for creating a lean capability framework.

\section{Lean Sustainability through Lean Capability Development}

When the term sustainability is used with respect to lean manufacturing, most think of how lean may support ecological preservation by reduction of waste of raw materials and energy supplies [8] or even the economic stability for an organization that provides opportunities for future growth and prosperity. Sustainability in this context generally refers to maintaining a balance between the exploitation of resources to fulfill the needs of today while ensuring protection of resources for survival in the future. The sustainability of lean itself is however also worth considering, both in terms of how to maintain momentum once initial pilots and "blitzes" are complete and on how members of the organization can actually develop their lean implementation capabilities. The former issue can be viewed as a relatively horizontal progression over time, as employees use lean tools for cost reduction in their daily work processes.

While the effects of these changes may increase as more people are involved and as lean is introduced to new areas, the activities and the capabilities of those performing them may remain relatively stable. On the other hand, as employees learn from their own and others' experiences, what may be viewed as a vertical progression would be expected to occur as well. Although both vertical and horizontal progression is implied in lean implementation models, there appears to be 
more focus on sustaining lean momentum in terms of spreading the concept to more organizational units than on ensuring lean development.

The issue of sustainability through vertical as well as horizontal development is central, albeit often implicit in many improvement initiatives. Researchers at the Center for Innovation Management (CENTRIM) at Brighton University addressed these issues specifically when developing what is referred to as the Continuous Improvement (CI) Maturity Model [9], which illustrates the gradual but steady development of CI through five stages of maturity through adoption of certain sets of behaviors that together build organizational capabilities. At the first level of the model, behavioral activities such as idea generation and problem-solving are implemented ad hoc, or what is often called "putting out fires". By creating systems, procedures, and processes in the organization, the CI development becomes more strategically oriented and structured until, at stage five maturity, a learning organization built on $\mathrm{CI}$ and organizational learning emerges.

There are distinct similarities between $\mathrm{CI}$ and lean-and in fact $\mathrm{CI}$ is a critical component in sustainable lean-and thus the model, with modifications to incorporate the specific characteristics of lean, should therefore be applicable to understanding how lean sustainability can be achieved through focus on capability development. Further, the development of lean as a concept parallels this maturation process, as described in the following section.

\section{Development of the concept of lean}

The origin of Lean can be traced back to Toyota in the 1950's, but did not become recognized until the beginning of the 1980's [10-14] and only first gained serious attention with the publication of The Machine that Changed the World [2]. Since its introduction, the concept of lean has developed considerably, beginning with an almost exclusive focus on shop floor workers in the automobile industry. In the 1990 's, the lean concept was extended not only to other industries, but also to much more than simply a "Toyota" and "Tools" orientation to a more comprehensive philosophy encompassing both strategic and organizational components. Hines et al. [15] suggests that this developmental progression of the lean concept can be described as occurring in four stages, similar to the four stage classification McGill and Slocum [16] use to portray the a company's progression towards becoming a learning organization. From a prescriptive focus on shop floor practices, tools and techniques applied in production cells and lines for higher efficiency in the 1980'es to a widened focus on quality and Lean as a set of management practices in the early 90's, still mainly applied within the automotive industry.

This widened focus is also evident in a review of Lean constructs based on Lean practises by Shah and Ward [17]. Here practices such as focused factory production, process capability measurements and cellular manufacturing are introduced to such constructs in 1990. According to Hines et al.'s [16] review the scope of Lean conceptualizations increased to value streams and focus shifted to flow creation from mid 90's. Customer value as constituted by cost, quality and delivery was inherited from the automotive supplier sector. From 2000 onwards the Lean concept has 
involved a greater degree of contingency and the scope has increased to value systems with concepts such as demand chain management. Further, in their review of 12 lean production models, Paez et al. [18], allude to a developmental progression of the lean concept from being primarily technically-oriented to one that also more strongly emphasizes the human elements of lean during the past two decades. A movement from singular practices such as team work, multi-tasking, and autonomy over to human resource practices, involvement, and empowerment that contribute to establishment of a learning environment is seen in their review.

Although the central focus of the research conducted by Hines et al. [16] and to some extend that of Paez et al. [18] is on describing the developmental progression of the lean concept itself, they may also provide some insights into the type of developmental progression lean must undergo within a given company in order to steadily progress from the isolated use of tools to a more integrated lean philosophy that also encompasses an organizational learning perspective. Balle [19] only refers to leaders, but also addresses the importance of progressing from simple tools and methods for lean to be successful over time when he describes the continuous learning that must occur for leaders to meet three phases of challenges: an early aha experience that inspires leaders to hunt for low hanging fruits and which results in a preference for quick action (1), a shift to a more rigorous, systematic and structure approach to problem solving and investigations to meet more complex challenges (2) and finally the realization that the leaders cannot solve all problems themselves, but must involve other employees (3).

How precisely this progression occurs, what is needed to allow the progression, and how the leaders are to know when they have reached one level of lean development and must become equipped to meet the next phase is however not directly addressed by the author. What is needed then is a framework that allows a company to assess its current status of lean development as well as identify areas in need of improvement, based on concrete descriptions of behaviors, mechanisms, processes, etc. necessary for successful, enduring lean. In the next section, the subject of assessment with respect to lean and the development of lean capability is discussed.

\section{Assessing Lean}

Assessment tools are critical to successful lean implementation--or in the successful implementation of any world class manufacturing principles for that matter. Assessment tools have many functions, most important perhaps as a "roadmap" that illustrates the company's current status among its most important performance parameters. A good assessment is also invaluable in identifying opportunities for improvement and the parameters in which action plans should be designed. For an assessment tool to fulfil these functions, it must accurately reflect the nature and complexity of what is being assessed. Based on the previous discussion on the importance of both "horizontal" and "vertical" lean development, this means that a lean assessment tool must address two perspectives or dimensions, each of which encompass a number of variables. Specifically, it is proposed that a lean assessment 
tool must include the following: (1) A technical perspective, which reflects performance, methods, and tools in relationship to the given company's strategic "scope", as described by Hines et al [16]; and (2) an organizational perspective, which reflects management, organizational and human capabilities, culture, and learning.

In addition to being able to evaluate variables related to each of these perspectives, a lean assessment tool should be able to measure the relative balance between the two elements and the possible synergy created by focusing attention on both perspectives simultaneously. The majority of available assessment tools, however, address primarily or exclusively the technical perspective [17] and only a select few refer aspects of the progressive lean development emphasized here (i.e. elements associated with the organizational perspective). Of those tools that do include mention of the organizational perspective of lean [20-21], even fewer consider the balance between the two perspectives and the potential synergy between them [22-23]. Finally, there do not appear to be any lean assessment tools that incorporate both perspectives while still emphasizing the processes necessary for ensuring developmental progression of lean in the organization.

In the following section of the paper, the methods used to gather data relevant to the construction of a model which emphasizes lean capability development and can be used to assess both the technical and organizational aspects of lean are briefly presented.

\section{Methods}

The study described in this paper is taken from a larger research project aimed at implementing lean with emphasis on creating a positive psychological environment for employees and involves data collection through workshops, seminars, interviews and observations conducted with and in 12 large Danish organizations currently implementing lean and representing the industrial, administrative, and service sectors. The collected data are used in this paper to develop the foundation of a framework for describing and assessing lean implementation that focuses both on performance improvement through lean (i.e. a technical perspective) and the lean capability development (i.e. an organizational perspective) and the balance of these proposed here as necessary for ensuring lean sustainability.

The four authors of the paper have been involved in the data collection following an action research methodology, with active participation in discussions of the companies' experiences. Data have been verified continuously through active dialogue with the participants in the study.

The lean capability model, which is presented in the following section, is derived from descriptions of both actual experiences with lean in the companies and what key persons implementing lean define as an ideal state for lean that would support lean capability development and long term sustainability. The data were classified into the maturity levels according to group consensus and are considered consistent with the lean literature. Further, the developmental phases (i.e. maturity levels) included in the model can be theoretically and practically compared to those found in 
the CI Maturity Model [9] which has been subject to numerous empirical applications [24].

\section{Lean Capability Model}

Experiences from these companies suggest a number of common trends, especially in the beginning or immature phases of the lean implementation. Much time is spent in trying to build a shared understanding of the lean philosophy and how it impacts the individual and the organization. Initially, training is limited to project leaders ("experts") and focuses primarily on skill training once extended to the general workforce (i.e. shop floor workers or those who will be responsible for working with lean). Indications of lean maturity first become apparent as technological and organizational mechanisms become aligned with the strategic objectives to be fulfilled through lean. At this point, HR functions become critical. Characteristics of the five identified levels of maturity are described below:

1. Sporadic production optimization: This level is characterized by occasional rather random efforts at optimization in various organizational unites, but these activities are not planned or implemented on the basis of an overall strategy or a specific manufacturing philosophy. The optimization projects are typically led by experts with little to no general employee involvement. Organizational mechanisms and systems are not integrated with lean philosophy and/or lean objectives.

2. Basic lean understanding and implementation: Lean has now been chosen as the manufacturing philosophy that will serve as the basis for production control and optimization. The experts and general workforce have received basic training and pilot projects have been initiated in isolated unites within the organization for the purpose of experimenting with the individual lean tools and methods. Isolated mechanisms developed to support lean (e.g. reward and suggestion schemes, training)

3. Strategic lean interventions: The implementation of lean is now a part of the organization's strategy and projects and activities are planned on the basis of established goals and objectives. Knowledge of and practical experience with lean tools and methods as well as a lean philosophy are widely acknowledged and recognized at all levels of the organization, although initiatives are still primarily implemented according to an established plan. Satisfactory performance improvements are achieved. Specific HR systems (i.e. selection, compensation, training functions) are aligned with lean objectives to support lean goals.

4. Proactive lean culture: Lean activities occur continuously from all areas of the organization. To think and act lean has become a part of the daily work, and $\mathrm{CI}$ is more of a habit than a specific task, although efforts have not yet been made to extend these efforts outside of the organization's own boundaries. The practical understanding of lean tools and methods is quite high and these are used actively by all members of the organization to develop and implement performance improvements. All HR functions are 
aligned with lean objectives for the purpose of supporting long term sustainability. Focus on, e.g. career development via lean and extended developmental activities (e.g. external education).

5. Lean in the EME: The lean strategy is no longer just an internal strategy and its impact is visible in activities throughout the EME (Extended Manufacturing Enterprise) level. Lean activities are planned, implemented, and monitored across the EME's boundaries. Knowledge sharing and knowledge transfer are important components of the act ivies across the EME and organizational structures support inter-organizational network building.

\section{Discussion and Conclusion}

Many authors state that lean implementation efforts often fail to provide companies with the long term benefits promised in the literature. Generally there is agreement that successful sustainable lean involves more than the use of tools and methods and efforts should be made to support development of a lean culture, but there is little in the literature to serve as a "roadmap" for companies wishing to support this development. On the basis of a brief literature review and experiences in companies currently implementing lean, this paper presents an emerging framework describing five stages of lean capability development that are consistent with long term sustainability of lean. One of the important findings in this study was that Human Resource (HR) functions may play a critical role in supporting what is referred to here as "vertical" development of lean. Specifically, training and development targeted at learning and knowledge sharing, compensation and reward schemes, and focus on lean as a means towards career development may facilitate establishment of a lean culture that is sustainable. The importance of organizational learning is also emphasized by Emiliani [25], who proposes that a lean production philosophy provides excellent opportunities to couple personal growth and learning needs with organizational performance objectives. The paper contributes to both theory and practice by describing the stages of lean capability development that are necessary for sustained success with lean and suggesting a framework for assessing a company's current level of lean capability maturity.

\section{References}

1. J. P. Womack and D.T. Jones, Lean Thinking: Banish Waste and Create Wealth in Your Corporation, Simon and Schuster: New York, NY (1996)

2. James P. Womack, Daniel T. Jones, and Daniel Roos, The Machine that Changed the World, Harper-Collins: New York (1990).

3. B. Harrison, Lean and Mean: The Changing Landscape of Corporate Power in the Age of Flexibility, The Guildford Press: New York (1994).

4. P.Landsbergis, J. Cahill J, and P. Schnall, The Impact of Lean Production and Related New Systems of Work Organization on Worker Health., In. of Occupational Health Psych., 4(2), 108-30 (1999). 
5. J.K. Liker, The Tovota Way - 14 Management Principles from the World's greatest Manufacturer, McGrawhill: New York, NY (2004).

6. N. Kinnie, S. Hutchninson, J. Purcell, C. Rees, H. Scarbrough, and M. Terr, The People Management Implications of Leaner Working, Institute of Personnel Management, London (1996).

7. R.J. Radnor and R. Boaden, Developing an understanding of corporate anorexia, Journal of Operations \& Production Management, 24(4), 424-440 (2004).

8. T. H. Johnson, Sustainability and lean operations, Cost Management, 20 (2), 4046 (2006).

9. J. Bessant and S. Caffyn, High involvement innovation", Int 'l. Jn. of Technology Mgmt. 14(1), 7-28 (1997).

10. S. Shingo, Study of the Toyota Production Systems, Japan management Association, Tokyo (1981).

11. S. Shinko, Non-Stock Production: The Shinko System for Continuous Improvement, Productivity Press: Cambridge, MA (1988).

12. R. Schonberger, World Class Manufacturing - The Lessons of Simplicity Applied, The Free Press: New York, NY (1986).

13. Y. Monden, The Toyota Production System, Productivity Press: Portland (1983).

14. T Ohno., The Toyota Production System: Beyond Large-Scale Production, Productivity Press: Portland, OR (1988).

15. Peter Hines, Matthias Holwe, and Nick Rich, Learning to evolve - A review of contemporary lean thinking, Int'l. Jn. of Operations \& Production Mgmt. 24(10), 994- 1011 (2004).

16. M.E. McGill and J.W. Slocum, Unlearning the organisation, Organisational Dynamics, 22(2), 67-79 (1993).

17. Rachna Shah and Peter T. Ward, Lean manufacturing: context, practice bundles, and performance, Jn. of Operations Mgmt. 21, 129-149 (2003).

18. O. Paez, J. Dewees, A. Genaidy, S. Tuncel, W. Karwowski, and J. Zurada, The Lean Manufacturing Enterprise: An Emerging Sociotechnological System Integration, Human Factors and Ergonomics in Manufacturing, 14(3), 285-306 (2004).

19. M. Ballé, Lean attitude - Lean applications often fail to deliver the expected benefites but could the missing link for successful implementations be attitude?, Manufacturing Engineer, 84(2), 14-1 (2005).

20. Christer Karlsson and Pär Åhlström, Assessing changes towards lean production, Int'l. Jn. of Operations and Production Mgmt., 16(2), 24-41 (1996)

21. LESAT, LEAN ENTERPRISE SELF-ASSESSMENT TOOL, MIT and University of Warwick, www.lean.mit.edu, Version 1.0 (2001).

22. T. L. Doolen, M. E. Hacker, A Review of Lean Assessment in Organizations: An Exploratory Study of Lean Practices by Electronics Manufacturers, Jn. of Mfg. Systems, 24(1), 55-67 (2005).

23. R. Sawhney, and S. Chason, Human Behavior Based Exploratory Model for Successful Implementation of Lean Enterprise in Industry, Performance Improvement Quarterly, 18(2), 76-96 (2005)

24. Sarah Caffyn, Development of a continuous improvement self-assessment tool, International Journal of Operations \& Production Management, 19(11), 11381153 (1999).

25. M. L. Emiliani, Lean behaviors, Management Decision, 36(9), 615-631 (1998). 